\title{
Article
}

\section{Diet Therapy for Inflammatory Bowel Diseases: A Call to the Dining Table}

Limketkai, Berkeley N, Gordon, Morris, Mutlu, Ece A, De Silva, Punyanganie S and Lewis, James D

Available at http://clok.uclan.ac.uk/31168/

Limketkai, Berkeley N, Gordon, Morris ORCID: 0000-0002-1216-5158, Mutlu, Ece A, De Silva, Punyanganie S and Lewis, James D (2020) Diet Therapy for Inflammatory Bowel Diseases: A Call to the Dining Table. Inflammatory Bowel Diseases, 26 (4). pp. 510-514. ISSN 1078-0998

It is advisable to refer to the publisher's version if you intend to cite from the work. http://dx.doi.org/10.1093/ibd/izz297

For more information about UCLan's research in this area go to http://www.uclan.ac.uk/researchgroups/ and search for <name of research Group>.

For information about Research generally at UCLan please go to http://www.uclan.ac.uk/research/

All outputs in CLoK are protected by Intellectual Property Rights law, including Copyright law. Copyright, IPR and Moral Rights for the works on this site are retained by the individual authors and/or other copyright owners. Terms and conditions for use of this material are defined in the policies page.

\section{CLoK}

Central Lancashire online Knowledge www.clok.uclan.ac.uk

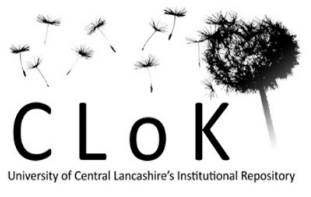




\section{Diet Therapy for Inflammatory Bowel Diseases: A Call to the Dining Table}

Berkeley N. Limketkai, MD, PhD, ${ }^{1}$ Morris Gordon, MBChB, MMed, PhD, ${ }^{2}$ Ece A. Mutlu, MD, MS, MBA, ${ }^{3}$ Punyanganie S. de Silva, MD, MPH, ${ }^{4}$ James D. Lewis, MD, MSCE ${ }^{5}$

${ }^{1}$ Vatche and Tamar Manoukian Division of Digestive Diseases, UCLA School of Medicine, Los Angeles, California, USA

${ }^{2}$ University of Central Lancashire, Preston, United Kingdom

${ }^{3}$ Section of Gastroenterology, Hepatology \& Nutrition, Rush University Medical Center, Chicago, Illinois, USA

${ }^{4}$ Center for Crohn's and Colitis, Division of Gastroenterology, Hepatology \& Endoscopy, Brigham and Women's Hospital, Boston, Massachusetts, USA

${ }^{5}$ Division of Gastroenterology, Perelman School of Medicine, University of Pennsylvania, Philadelphia, Pennsylvania, USA.

\section{Corresponding Author:}

Berkeley N. Limketkai, MD, PhD

Manoukian Division of Digestive Diseases

UCLA School of Medicine

Los Angeles, California

E-mail: berkeley.limketkai@gmail.com

Authorship: All authors contributed to the conceptual design, drafting, critical revision, and approval of the manuscript.

Funding: None

Conflicts of Interest: None

Keywords: Diet, evidence, inflammation, symptoms 


\title{
SUMMARY
}

The article comments on the recent systematic review and meta-analysis of dietary interventions for Crohn's disease and ulcerative colitis. We discuss the pitfalls of current data and emphasize the importance of further research into diets and inflammatory bowel diseases.

\author{
ABSTRACT \\ There is vigorous interest among patients, caregivers, clinicians, and scientists to discover \\ useful dietary interventions for inflammatory bowel diseases (IBD). Through the Cochrane \\ Collaboration, we recently performed a systematic review and meta-analysis of dietary \\ interventions for the induction or maintenance of remission in Crohn's disease (CD) and \\ ulcerative colitis (UC) to assess the latest state of research. The current quality of evidence was \\ formally graded to be low or very low for various methodological reasons, such as small sample \\ sizes, heterogeneity among studies, and incomplete reporting. There are nonetheless emerging \\ observational studies that progressively advance our knowledge and provide hope for a role of \\ diet among traditional therapies to improve inflammation and symptoms. Further investments \\ and concerted efforts in research are needed to significantly move the needle in identifying \\ effective dietary therapies for IBD.
}




\section{INTRODUCTION}

The hypothesis that pathogenesis of inflammatory bowel diseases (IBD) is strongly influenced by non-genetic (environmental) factors has fueled intense interest in identifying potentially modifiable factors for the prevention and treatment of IBD. Since one of the several pillars of this hypothesis is dysbiotic alterations in the gut microbiome, research efforts have explored diverse microbiome manipulation approaches, such as the use of prebiotics, ${ }^{1}$ probiotics,${ }^{2-5}$ fecal microbiota transplantation, ${ }^{6}$ and diet as potential therapeutic strategies. The premise of these approaches presumes that reconfiguration of the microbiome toward a more "eubiotic" or less pro-inflammatory profile would lead to a reduction of intestinal inflammation. The premise of dietary manipulation takes a step further by hypothesizing that the reduced exposure to proinflammatory antigens (e.g., additives, emulsifiers, omega- 6 fatty acids) and/or addition of antiinflammatory constituents (e.g., omega-3 fatty acids, curcumin) in consumed foods would tip the balance toward a lower inflammatory profile. The feasibility of dietary therapies to influence intestinal inflammation is illustrated by the efficacy of exclusive enteral nutrition (EN) to induce remission in Crohn's disease (CD), where society guidelines even recommend the use of exclusive EN as a steroid-sparing first-line therapy in children with active CD. ${ }^{7-10}$ Paradoxically, exclusive EN initially exacerbates dysbiosis by narrowing gut bacterial diversity and reducing gut concentrations of presumably protective bacteria, although dysbiosis eventually improves as inflammation resolves. ${ }^{11,12}$ These observations suggest that the mechanisms of dietary therapies' benefits may include other factors in addition to the microbiome. Moreover, they reflect the complex interactions between diet and microbes, whereby bacterial diversity alone may be too simple of a marker of "microbial health".

There is currently vigorous interest among patients, caregivers, clinicians, and scientists to discover useful dietary interventions to either serve as primary or adjunctive therapy to optimize treatment response to traditional immunosuppressive agents. There is a dizzying array of anecdotes, conjectures, and misinformation about effective diets for IBD circulating on the 
Internet. Many of the recommendations extrapolate concepts from other gastrointestinal disorders, such as irritable bowel syndrome, but are generally unproven to reduce inflammation. ${ }^{13}$ The patients' need to search and share dietary guidance within the online community is understandable, although there is an equally great need to address knowledge gaps and to differentiate fact from fiction.

\section{CURRENT STATE OF EVIDENCE}

A recent Cochrane systematic review and meta-analysis of dietary interventions for the induction or maintenance of remission in $\mathrm{CD}$ or ulcerative colitis $(\mathrm{UC})^{14}$ has been published. All randomized controlled trials (RCTs) of dietary interventions for IBD through January 2019 were carefully reviewed, among which 18 RCTs with 1878 participants met criteria for quantitative and qualitative analyses. A description of the diets studied is available in the review. ${ }^{13}$

For, CD, there were 6 studies evaluating 5 diet variants for the induction of remission. A highly restricted organic diet (relative risk [RR] 1.00; 95\% confidence interval [CI] $0.39-2.53 ; 1$ study with 18 participants) and diets defined by a reduction in refined carbohydrates (RR 7.20; $95 \% \mathrm{Cl} 0.53-97.83 ; 1$ study with 7 participants), microparticles (RR 3.13; 95\% Cl $0.22-43.84$; 2 studies with 103 participants; $I^{2}=73 \%$ ), or calcium (RR 1.24; 95\% Cl $0.67-2.29 ; 1$ study with 83 participants) did not confer added benefit over generic control diets for the achievement of clinical remission. On the other hand, a symptom-guided elimination diet was superior to "conventional nutritional advice" for achieving clinical remission in mild CD (RR 20.0; $95 \% \mathrm{Cl}$ $1.27-315.40 ; 1$ study with 51 participants). Only the study using a highly restricted organic diet evaluated inflammatory activity as an outcome and found no difference in serologic or endoscopic markers. For the maintenance of remission, there were 7 studies that evaluated 4 types of exclusion diets. Diets with low refined carbohydrates (RR 1.04; $95 \% \mathrm{Cl} 0.87-1.25 ; 3$ studies with 567 participants; $I^{2}=35 \%$ ), symptom-provoking foods (RR $0.53 ; 95 \% \mathrm{Cl} 0.28$ $1.01 ; 2$ studies with 98 participants; $I^{2}=54 \%$ ), red or processed meats (RR $1.03 ; 95 \% \mathrm{Cl} 0.85-$ 
1.26; 1 study with 214 participants), or a combination of the above (RR $0.11 ; 95 \% \mathrm{Cl} 0.01-$ 1.76; 1 study with 54 participants) were not superior than generic control diets for reducing clinical relapse. Other than two studies that measured C-reactive protein (CRP) and erythrocyte sedimentation rate (ESR) concentrations, there were no outcomes of inflammatory disease activity.

For UC, one study evaluated the symptoms-guided diet for the induction of remission and found no benefit over an unaltered diet for induction of remission (RR $8.25 ; 95 \% \mathrm{Cl} 0.50-$ 136.33; 21 participants). There was no clear endoscopic or histologic improvement either. Four studies evaluated 3 diets for the maintenance of remission. There was no difference in clinical relapse rates when comparing an anti-inflammatory diet (RR 1.25; 95\% Cl $0.42-3.70 ; 1$ study with 28 participants), carrageenan-free diet (RR $0.50 ; 95 \% \mathrm{Cl} 0.15-1.64 ; 1$ study with 15 participants), or milk-free diet (RR $0.83 ; 95 \% \mathrm{Cl} 0.60-1.15 ; 2$ studies with 77 participants; $\mathrm{I}^{2}=$ $0 \%)$ with generic control diets.

The certainty of evidence for each of these evaluated outcomes were formally graded as being low or very low, primarily due to small sample sizes, heterogeneity in the methods, and incomplete reporting on clinical trial quality measures (e.g., how blinding and analyses were performed). This currently would mean that there are no actionable dietary recommendations based on the available evidence. However, the likelihood is that these findings will change with more research. Notably, this is not the same as demonstrating lack of efficacy in any of the individual studies, rather, that the analyses demonstrate no definite conclusions can be reached based on the available evidence to date. It is worth noting that the well-established methods to judge bias, quality and certainty, these do not consider the quality of reporting of the intervention itself. This is not of real significance in studies of medications, but in the context of diet, the detail of the diet is vital as the application may vary greatly for similarly named diets and "300 $\mathrm{mg}$ " of diet cannot be prescribed. 
While this systematic review used stringent criteria to formally assess the state of current RCT data for dietary interventions to treat IBD, non-randomized observational studies represent emerging sources of information that can potentially provide further insight into other diet candidates that need further exploration. Similar to the diets studied in published RCTs, variants of exclusion diets have been popular, such as the Specific Carbohydrate Diet (SCD),${ }^{15}$ glutenfree diet, and Crohn's Disease Exclusion Diet (CDED) ${ }^{16}$ Early observational evidence has shown the SCD and CDED to potentially be helpful for inducing remission in $C D .{ }^{17-19} \mathrm{An}$ extension to the theme of exclusion diets are those that incorporate nutrients with putative antiinflammatory or immunomodulatory properties; prototypes of such diets include the antiinflammatory diet and Mediterranean diet. ${ }^{20,21}$ These diets limit the consumption of refined carbohydrates and saturated fats, while leveraging potentially beneficial phytonutrients in fruits and vegetables, as well as oils (e.g., olive oil, fish oil) rich in omega-3 polyunsaturated fats. Given the established benefit of EN for induction of remission in CD, other diets (e.g., CDTREAT) attempt to recapitulate the microbiome effects of of $E N,{ }^{22}$ or combine them with partial EN. ${ }^{16}$

While some of these observational studies show the potential of several diets for the treatment of IBD, there is a strong caveat about prematurely attributing efficacy using lowquality evidence. For instance, a pilot RCT in 20 patients showed a significant difference in the change and resulting CDAl after 4 months, when comparing those who received the low microparticle diet versus a control diet. ${ }^{23}$ There was also an impressive difference of $70 \%$ versus $0 \%$ achieving clinical remission, respectively. However, when the investigators launched a similar follow-up trial with a more robust sample size of 83 participants, they found no difference between the low versus normal microparticle diets. ${ }^{24}$ This example illustrates the caution in overinterpreting small studies. Nonetheless, these studies serve a critical role for informing subsequent higher quality investigation, such as the CDED, PRODUCE (NCT03301311), and DINE-CD (NCT03058679) trials. After our systematic review, an RCT was 
published evaluating the efficacy of CDED coupled with partial EN for the induction of remission in 78 children with mild-to-moderate CD. ${ }^{16}$ Participants were initially randomized to either receive exclusive EN or 50\% CDED (50\% partial EN). There was no difference in 6-week clinical response between the groups, suggesting similarity in efficacy. Participants in the exclusive EN group thereafter transitioned to $75 \%$ unrestricted diet (25\% partial EN), while those in the CDED group transitioned to $75 \%$ CDED (25\% partial EN). By week 12, participants receiving CDED had greater sustained remission than those following an unrestricted diet. The other ongoing trials were designed to rigorously compare the effectiveness of the SCD with a modified SCD (PRODUCE) or a Mediterranean-style diet (DINE-CD) for the induction of remission in IBD.

\section{CHALLENGES WITH RESEARCH}

The primary limitations with the current evidence on dietary interventions in IBD are their methodological design and execution. The sample sizes of available RCTs have been exceedingly low with a median among the studies of 38 participants. While it is possible that some of the diets may be effective for the induction or maintenance of remission, the trials' inability to demonstrate significant benefit could realistically stem from having inadequate power to detect a difference between the treatment and control diets. Furthermore, heterogeneity in baseline disease activity, lack of clarity on what diets were consumed prior to enrollment, and the presence of other potential confounders would have a stronger influence on bias in small studies despite use of randomization to "homogenize" treatment populations.

There was great heterogeneity among dietary interventions studied, generally leaving only 1 or 2 trials per type of diet and diminishing the benefit of meta-analyses for overcoming small sample sizes by aggregating similar studies in an effort to generate specific guidance for clinicians and patients. This heterogeneity of interventions reflects the disparities in individual research efforts, transient research interest in each diet, and our current lack of understanding 
or agreement on what constitutes the ideal diet. We do not yet understand what constitutes an "optimal dose" of anti-inflammatory nutrients. Linked to this is the assumption that the description of similar diets may not actually accurately match what the patient is actually given or indeed complies with.

The methods of dietary intervention, including assessment and assurance that the right foods within the designated diets were selected or even consumed (compliance) are unclear. These concerns are highly prevalent in dietary intervention studies where, unless the investigators provide the meals to participants, maintaining them within the strict parameters of what define the dietary intervention can be practically very challenging.

The outcomes selected for the available RCTs have predominantly focused on symptom scores to define clinical remission. There is a general lack of objective markers of inflammatory activity, such as endoscopic or histologic outcomes, or dysbiosis. Symptoms can improve with dietary modification, particularly learned avoidance of symptom-provoking foods; however, the impact of these modifications on inflammation, a central mechanism that drives symptoms and the progression of disease, would still be unknown. The Crohn's Disease Activity Index (CDAI) used in many studies is strongly dependent on reported symptoms, so it is not surprising that participants who avoided symptoms-provoking foods would experience fewer symptoms.

The risk of bias assessment showed that many of the trials had a high or unclear risk of bias. The high risk of bias predominantly involved inadequate blinding of participants and personnel, although this may be a general limitation of dietary interventions, as the types, tastes, and textures of food would be difficult to homogenize across treatment arms. The recently devised update to the Cochrane risk of bias tool may allow such elements to not be rated as high risk in the future. However, much of the unclear risk of bias stemmed from inadequate reporting on how randomization was performed. This oversight could have been easily circumvented and given their key role in ensuring adequate trial design, this is a significant issue with the evidence base. 
In an attempt to overcome these methodologic challenges, the DINE-CD trial has a robust sample size target of 194 participants, provides prepared meals to minimize heterogeneity of consumed foods, and employs fecal calprotectin as a surrogate marker of inflammation in addition to reported symptoms. Nonetheless, despite the rigor in its study design, the interventions are not blinded and there are no endoscopic or histologic data. These limitations were not due to oversight, but illustrate the added challenges of balancing cost and practicality when designing high-quality studies, practicality, and the need for a high-quality study.

\section{CALL TO THE ROUND [DINING] TABLE}

The major gastroenterological societies are relatively silent on the role of dietary therapies in IBD, except for recommendations on the use of exclusive EN for CD (Table 1). The current lack of evidence on dietary therapies for IBD does not necessarily signify that they have no role in helping control inflammation. Given the diet's and gut microbiome's known effects in altering inflammation at the molecular level, the potential to eventually identify diets that macroscopically affect inflammation endoscopically and histologically is strong. We just do not yet know which diets and in what context they would be helpful, such as in prevention or treatment of IBD. The interactions between food and biology are immensely complex; while EN is recommended for the induction of remission in children with active $C D$, it is not efficacious for UC. This disparity demonstrates that dietary alteration of pro-inflammatory and anti-inflammatory substances does not alone explain nutritional therapies' effect on inflammation. Furthermore, studies with children on EN demonstrate changes in the microbiota that may be considered further than normal despite the clinical improvements seen. These findings suggest that dietary interventions have complex and multiple unexpected effects that are yet to be fully explored. There are also very

likely patient-specific factors that influence the potential efficacy of dietary therapy. It is also still unclear whether diet may serve as a primary or adjunctive therapy. Would it be an aid for 
improving overall response to a backbone of pharmacologic immunosuppression or a method to permit partial therapy de-escalation? The position of dietary therapy in the overall treatment algorithm and the clinical and/or genetic factors that determine in whom these would be appropriate (personalized nutrition) have yet to be defined. As many of the dietary studies have been done in children, the translatability of these findings to the adult population, or vice versa, is also unknown.

This is therefore a call to arms - or, to the dining table - that a concerted effort to advance research on dietary therapies in IBD is needed. The dearth of RCTs, most dating back many years and some published only as abstracts, reflects the relative lack of attention on this area of research and the challenges faced by researchers to engage in well-designed trials. There is a need for interest and investment in these costly endeavors. There is a need to push forward with research programs on particular diets to progressively accumulate data.

Unfortunately, the majority of the RCTs and diets in the systematic review never moved beyond the first small study. Even the symptoms-guided diet that was reportedly effective at improving symptoms in CD was never published. With many small studies with heterogeneous diets, the data needed to demonstrate dietary efficacy - if true - would not be expected to be generated. The methodologic challenges with many of the available dietary intervention studies also need to be addressed and improved. Lewis et al provide valuable guidance on key considerations when designing such trials. ${ }^{25}$

The patient community participates in research trials to help build evidence that would hopefully benefit them and their peers in the future. Funding agencies and philanthropists contribute resources in hopes of helping the discovery of important and innovative therapies. Unlike drug development trials, lack of funding opportunities presents a major challenge unique to diet studies. In the absence of such funding, the state of nutrition research in IBD will continue to progress at a glacial pace. It behooves the research community to make use of 
these investments to build on the growing foundation of knowledge and eventually generate high-quality evidence to show whether and what diets may be used to treat IBD or not.

Ensuring that such research is performed in a manner that will increase the likelihood of impact is vital. We believe that international societies need to coordinate a strategy based on current evidence of all forms. Key candidate therapies should be agreed on and a public unified call made to encourage research focused in these areas. These discussions must include patient advocate societies and charities, as patient views on the tolerability, cost, and interest in diets will be vital in gaining momentum for investigation. So, we end with a call not just to the dining table, but to the virtual round table to bring forth the much needed highly relevant evidence in this vital area of importance to our patients and clinicians alike. 


\section{REFERENCES}

1. Laurell A, Sjoberg K. Prebiotics and synbiotics in ulcerative colitis. Scand J Gastroenterol. 2017;52(4):477-85.

2. Butterworth AD, Thomas AG, Akobeng AK. Probiotics for induction of remission in Crohn's disease. Cochrane Database Syst Rev. 2008(3):CD006634.

3. Rolfe VE, Fortun PJ, Hawkey CJ, Bath-Hextall F. Probiotics for maintenance of remission in Crohn's disease. Cochrane Database Syst Rev. 2006(4):CD004826.

4. Naidoo K, Gordon M, Fagbemi AO, Thomas AG, Akobeng AK. Probiotics for maintenance of remission in ulcerative colitis. Cochrane Database Syst Rev. 2011(12):CD007443.

5. Mallon P, McKay D, Kirk S, Gardiner K. Probiotics for induction of remission in ulcerative colitis. Cochrane Database Syst Rev. 2007(4):CD005573.

6. Imdad A, Nicholson MR, Tanner-Smith EE, et al. Fecal transplantation for treatment of inflammatory bowel disease. Cochrane Database Syst Rev. 2018;11:CD012774.

7. Ruemmele FM, Veres G, Kolho KL, et al. Consensus guidelines of ECCO/ESPGHAN on the medical management of pediatric Crohn's disease. J Crohns Colitis. 2014;8(10):1179-207.

8. Mowat C, Cole A, Windsor A, et al. Guidelines for the management of inflammatory bowel disease in adults. Gut. 2011;60(5):571-607.

9. Forbes A, Escher J, Hebuterne X, et al. ESPEN guideline: Clinical nutrition in inflammatory bowel disease. Clin Nutr. 2017;36(2):321-47.

10. Critch J, Day AS, Otley A, et al. Use of enteral nutrition for the control of intestinal inflammation in pediatric Crohn disease. J Pediatr Gastroenterol Nutr. 2012;54(2):298305.

11. Gerasimidis K, Bertz M, Hanske L, et al. Decline in presumptively protective gut bacterial species and metabolites are paradoxically associated with disease improvement in pediatric Crohn's disease during enteral nutrition. Inflamm Bowel Dis. 2014;20(5):86171.

12. Lewis JD, Chen EZ, Baldassano RN, et al. Inflammation, Antibiotics, and Diet as Environmental Stressors of the Gut Microbiome in Pediatric Crohn's Disease. Cell Host Microbe. 2015;18(4):489-500.

13. Hou JK, Lee D, Lewis J. Diet and inflammatory bowel disease: review of patient-targeted recommendations. Clin Gastroenterol Hepatol. 2014;12(10):1592-600.

14. Limketkai BN, Iheozor-Ejiofor Z, Gjuladin-Hellon T, et al. Dietary interventions for induction and maintenance of remission in inflammatory bowel disease. Cochrane Database Syst Rev. 2019;2:CD012839.

15. Gottschall EG. Breaking the Vicious Cycle: Intestinal Health through Diet. Ontario, Canada: Kirkton Press; 1994.

16. Levine A, Wine E, Assa A, et al. Crohn's Disease Exclusion Diet Plus Partial Enteral Nutrition Induces Sustained Remission in a Randomized Controlled Trial. Gastroenterology. 2019.

17. Obih C, Wahbeh G, Lee D, et al. Specific carbohydrate diet for pediatric inflammatory bowel disease in clinical practice within an academic IBD center. Nutrition. 2016;32(4):418-25.

18. Cohen SA, Gold BD, Oliva S, et al. Clinical and mucosal improvement with specific carbohydrate diet in pediatric Crohn disease. J Pediatr Gastroenterol Nutr. 2014;59(4):516-21.

19. Sigall-Boneh R, Pfeffer-Gik T, Segal I, Zangen T, Boaz M, Levine A. Partial enteral nutrition with a Crohn's disease exclusion diet is effective for induction of remission in 
children and young adults with Crohn's disease. Inflamm Bowel Dis. 2014;20(8):1353-

60.

20. Olendzki BC, Silverstein TD, Persuitte GM, Ma Y, Baldwin KR, Cave D. An antiinflammatory diet as treatment for inflammatory bowel disease: a case series report. Nutr J. 2014;13:5.

21. Marlow G, Ellett S, Ferguson IR, et al. Transcriptomics to study the effect of a Mediterranean-inspired diet on inflammation in Crohn's disease patients. Hum Genomics. 2013;7:24.

22. Svolos V, Hansen R, Nichols B, et al. Treatment of Active Crohn's Disease With an Ordinary Food-based Diet That Replicates Exclusive Enteral Nutrition. Gastroenterology. 2019;156(5):1354-67 e6.

23. Lomer MC, Harvey RS, Evans SM, Thompson RP, Powell JJ. Efficacy and tolerability of a low microparticle diet in a double blind, randomized, pilot study in Crohn's disease. Eur J Gastroenterol Hepatol. 2001;13(2):101-6.

24. Lomer MC, Grainger SL, Ede R, et al. Lack of efficacy of a reduced microparticle diet in a multi-centred trial of patients with active Crohn's disease. Eur J Gastroenterol Hepatol. 2005;17(3):377-84.

25. Lewis JD, Albenberg L, Lee D, Kratz M, Gottlieb K, Reinisch W. The Importance and Challenges of Dietary Intervention Trials for Inflammatory Bowel Disease. Inflamm Bowel Dis. 2017;23(2):181-91. 